\title{
dynamical evolution of scalar perturbation in Hořava-Lifshitz black-hole spacetimes
}

\author{
Chikun Ding, Songbai chen, and Jiliang Jing* \\ Institute of Physics and Department of Physics, \\ Hunan Normal University, Changsha, \\ Hunan 410081, People's Republic of China \\ and \\ Key Laboratory of Low Dimensional Quantum Structures \\ and Quantum Control (Hunan Normal University), \\ Ministry of Education, P. R. China.
}

\begin{abstract}
We study the dynamical evolution of a massless scalar perturbation in the Hořava-Lifshitz blackhole spacetimes with the coupling constants $\lambda=\frac{1}{3}, \lambda=\frac{1}{2}$ and $\lambda=3$, respectively. Our calculation shows that, for the three cases, the scalar perturbations decay without any oscillation in which the decay rate imprints the parameter of the Horava-Lifshitz black hole. The results are quite different from those in the Schwarzschild AdS black hole and can help us understand more about the Hořava-Lifshitz gravity.
\end{abstract}

Keywords: Hořava-Lifshitz black hole, Scalar perturbation, Dynamical evolution.

PACS numbers: 04.70.Dy, 04.62.+v, 97.60.Lf

* Corresponding author, Electronic address: jljing@hunnu.edu.cn 


\section{INTRODUCTION}

Einstein's general relativity is increasingly important in the modern physics, especially in the frontiers of very large distance scales including astrophysics and cosmology. However, it is proved to be non-renormalizable by quantum field theories. Therefore, numerous attempts of its modification have appeared in the literatures. Recently, Hořava [1] proposes a power counting renormalizable gravity theory in four dimensions, which admits the Lifshitz scale-invariance in time and space rather than Lorentz invariant theory of gravity at ultraviolet level. In the infrared limit, the higher derivative terms do not contribute and Hořava-Lifshitz gravity reduces to standard general relativity. Thus, Hořava-Lifshitz gravity can be regarded as an ultra-violet complete theory of general relativity.

Due to these novel features, Hořava-Lifshitz gravity theory has been intensively investigated |2, 3, 4, 5, 6, 7, 8, 9, 10, 11] and its cosmological applications have been studied [12, 13, 14, 15, 16, 17, 18]. Some static spherically symmetric black hole solutions with non-vanishing cosmological constant have been found in Hořava-Lifshitz theory [19, 20, 21, 22, 23] and the associated thermodynamic properties with those black hole solutions have been investigated [24, 25, 26, 27]. The potentially observable properties of black holes in the deformed Hořava-Lifshitz gravity were considered by using the gravitational lensing [28, 29], quasinormal modes [30] and the accretion disk [31]. These results could provide a way to distinguish the deformed HořavaLifshitz theory from standard general relativity. However, the observable properties of black hole in the Hořava-Lifshitz gravity with detailed balance condition is open in my knowledge. The main purpose of this paper is to study the dynamical evolution of a massless scalar perturbation in the Hořava-Lifshitz black-hole spacetime. We consider three special cases in which the coupling constant $\lambda=1 / 3, \lambda=1 / 2$ and $\lambda=3$ and to see whether there exists some new features in the dynamical evolution of the perturbation.

The paper is organized as follows. In Sec.II we review in brief the black hole solution in the Hořava-Lifshitz gravity. In Sec.III, we adopt to the Horowitz-Hubeny approach to study the evolution of a massless scalar perturbation in these background. In Sec. IV, we present our numerical results and make a comparison between the properties of the scalar perturbation in Hořava-Lifshitz black hole and Schwarzschild AdS black hole. We summarize and discuss our conclusions in the last section. 


\section{BLACK HOLES IN HOŘAVA-LIFSHITZ GRAVITY}

The four-dimensional metric in the ADM formalism can be expressed as [32]

$$
d s_{A D M}^{2}=-N^{2} d t^{2}+g_{i j}\left(d x^{i}-N^{i} d t\right)\left(d x^{j}-N^{j} d t\right)
$$

and the Hořava gravity action reads $[1,20]$

$$
\begin{aligned}
S_{H L} & =\int d t d^{3} x\left(\mathcal{L}_{0}+\mathcal{L}_{1}\right), \\
\mathcal{L}_{0} & =\sqrt{g} N\left\{\frac{2}{\kappa^{2}}\left(K_{i j} K^{i j}-\lambda K^{2}\right)+\frac{\kappa^{2} \mu^{2}\left(\Lambda_{W} R-3 \Lambda_{W}^{2}\right)}{8(1-3 \lambda)}\right\}, \\
\mathcal{L}_{1} & =\sqrt{g} N\left\{\frac{\kappa^{2} \mu^{2}(1-4 \lambda)}{32(1-3 \lambda)} R^{2}-\frac{\kappa^{2}}{2 w^{4}}\left(C_{i j}-\frac{\mu w^{2}}{2} R_{i j}\right)\left(C^{i j}-\frac{\mu w^{2}}{2} R^{i j}\right)\right\},
\end{aligned}
$$

where $C_{i j}$ is the Cotton tensor

$$
C^{i j}=\epsilon^{i k \ell} \nabla_{k}\left(R_{\ell}^{j}-\frac{1}{4} R \delta_{\ell}^{j}\right)=\epsilon^{i k \ell} \nabla_{k} R_{\ell}^{j}-\frac{1}{4} \epsilon^{i k j} \partial_{k} R .
$$

Taking $N^{i}=0$, the spherically symmetric solutions are [19, 20, 21, 22, 23, 24, 25, 26]

$$
d s_{S S}^{2}=-\tilde{N}^{2}(r) f(r) d t^{2}+\frac{d r^{2}}{f(r)}+r^{2}\left(d \theta^{2}+\sin ^{2} \theta d \varphi^{2}\right)
$$

with

$$
\begin{aligned}
& f=1+r^{2}-m r^{p_{ \pm}(\lambda)}, \quad p_{ \pm}(\lambda)=\frac{2 \lambda \pm \sqrt{6 \lambda-2}}{\lambda-1}, \\
& \tilde{N}=r^{q_{ \pm}(\lambda)}, \quad q_{ \pm}(\lambda)=-\frac{1+3 \lambda \pm 2 \sqrt{6 \lambda-2}}{\lambda-1}
\end{aligned}
$$

where $m$ is an integration constant related to the mass of the black hole and $\lambda \geq \frac{1}{3}$. Hereafter we choose the signs of $p(\lambda)$ and $q(\lambda)$ are negative because $p_{+}(\lambda)$ and $q_{+}(\lambda)$ are meaningless for $\lambda=1$. Moreover, we consider only three special cases $\lambda=\frac{1}{3}, \lambda=\frac{1}{2}$ and $\lambda=3$ because in these cases the values of $p(\lambda)$ and $q(\lambda)$ are integers, which are convenient for us to study the dynamical evolution of the scalar perturbations in the following calculations. In the table I, we list the $f(r), \tilde{N}(r)$ and Hawking temperature of Hořava-Lifshitz black holes for $\lambda=\frac{1}{3}, \lambda=\frac{1}{2}$ and $\lambda=3$ respectively. For the case $\lambda=\frac{1}{3}$, although the expression of $f(r)$ is the same as that of Schwarzschild AdS black hole, its Hawking temperature is not equal to that of Schwarzschild AdS black hole since $\tilde{N}(r)=r^{3}$ in the Hořava-Lifshitz black hole. This means that the thermodynamical properties of Hořava-Lifshitz black holes are completely different from those in the usual Schwarzschild AdS black hole. The similar discussions for $\lambda=\frac{1}{2}$ and $\lambda=3$ has been done in Refs. 24, 25, 26, 27]. 


\begin{tabular}{cccc}
\hline \hline$\lambda$ & $f(r)$ & $\tilde{N}(r)$ & $T_{H}$ \\
\hline$\frac{1}{3}$ & $1-\frac{m}{r}+r^{2}$ & $r^{3}$ & $\frac{1}{4 \pi} r_{+}^{2}\left(3 r_{+}^{2}+1\right)$ \\
$\frac{1}{2}$ & $1-m+r^{2}$ & $r$ & $\frac{1}{2 \pi} r_{+}^{2}$ \\
3 & $1-m r+r^{2}$ & $r^{-1}$ & $\frac{1}{4 \pi r_{+}^{2}}\left(r_{+}^{2}-1\right)$ \\
\hline
\end{tabular}

TABLE I: The metric functions $f(r), \tilde{N}(r)$ and Hawking temperature of Hor̆ava-Lifshitz black holes for $\lambda=\frac{1}{3}, \lambda=\frac{1}{2}$ and $\lambda=3$ respectively.

\section{DYNAMICAL EVOLUTION OF SCALAR PERTURBATION IN HOŘAVA-LIFSHITZ BLACK HOLES}

The evolutional equation for a massless scalar perturbation is

$$
\frac{1}{\sqrt{-g}} \partial_{\mu}\left(\sqrt{-g} g^{\mu \nu} \partial_{\nu}\right) \Phi(t, r, \theta, \varphi)=0
$$

Setting $\Phi(t, r, \theta, \varphi)=e^{-i \omega t} R(r) Y_{l m}(\theta, \varphi) / r$ and defining the tortoise coordinate

$$
d r_{*}=1 / F(r) d r
$$

where $F(r)=r^{\xi / 2} f(r)$ with $\xi=6,2,-2$ for $\lambda=\frac{1}{3}, \lambda=\frac{1}{2}$ and $\lambda=3$, we find the radial equation for the scalar perturbation in the Hořava-Lifshitz black-hole spacetime reads

$$
\frac{d^{2} R(r)}{d r_{*}^{2}}+\left(\omega^{2}-V\right) R(r)=0
$$

with

$$
V=F(r)\left[\frac{1}{r} \frac{d F(r)}{d r}+\frac{l(l+1)}{r^{2}} r^{\xi / 2}\right]
$$

We know from Fig. (11) that, for the cases $\lambda=\frac{1}{3}$ and $\lambda=\frac{1}{2}$, the effective potential $V(r)$ are divergent at infinity, which is similar to that in Schwarzschild AdS black hole. For the case $\lambda=3$, the effective potential $V(r)$ approaches to the constant 1.

Let us now use the Horowitz-Hubeny approach [33, 34] to study the evolution of the scalar perturbations in the Hořava-Lifshitz black holes above. Due to the special asymptotical properties of their effective potentials $V(r)$ at infinity, we take the following boundary condition

$$
R(r) \sim\left\{\begin{array}{c}
e^{-i \omega r_{\star}}, \quad r \rightarrow r_{+}, \\
0, \quad r \rightarrow+\infty,
\end{array}\right.
$$

which ensures that the solution is purely ingoing waves at the event horizon and is not divergent at infinity at the same time. 

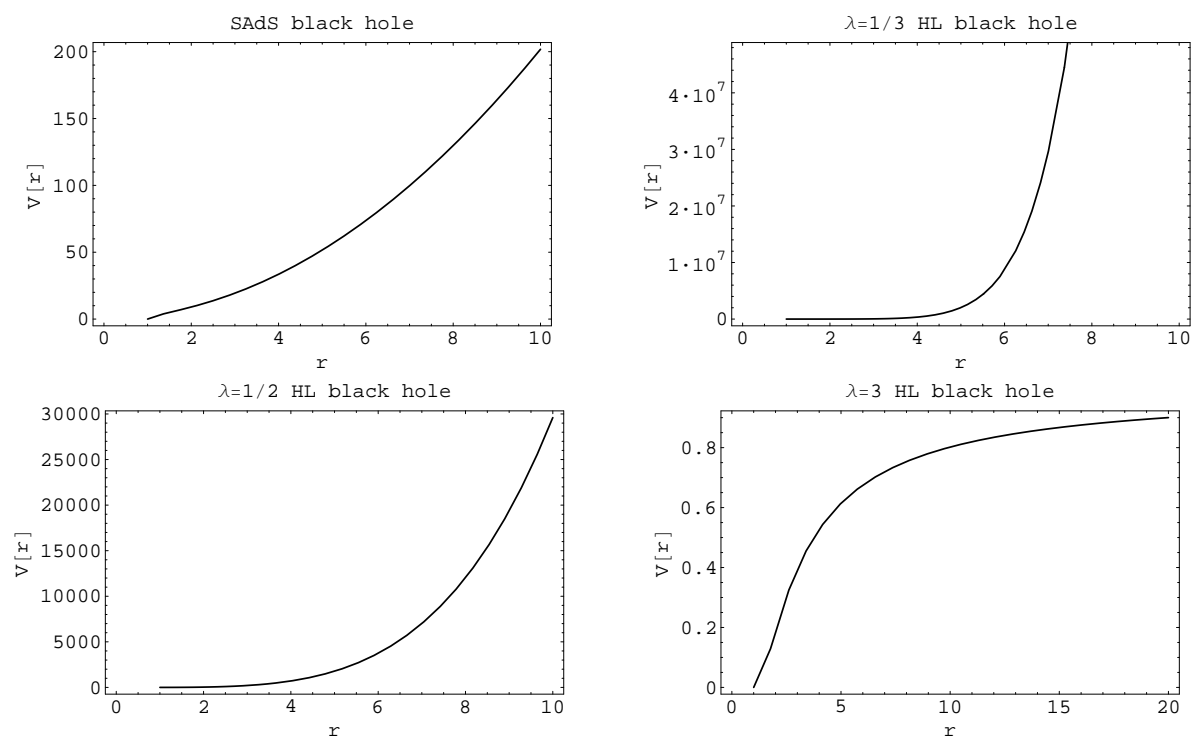

FIG. 1: Variety of the effective potential $V(r)$ with $r$ for Schwarzschild AdS black hole and the Hořava-Lifshitz black holes with $\lambda=\frac{1}{3}, \frac{1}{2}$ and 3 , respectively. Here we set $l=0$ and $r_{+}=1$.

Writing $R(r)=e^{-i \omega r_{*}} \phi$, Eq. (10) can be rewritten as

$$
\frac{1}{r^{\xi / 2}}\left[F \frac{d^{2} \phi}{d r^{2}}+\left(\frac{d F}{d r}-2 i \omega\right) \frac{d \phi}{d r}-\frac{1}{r} \frac{d F}{d r} \phi\right]-\frac{l(l+1)}{r^{2}} \phi=0
$$

Setting $r=\frac{1}{x}$ to change the physical region of $r_{+}<r<+\infty$ into $x_{+}>x>0$, and then using $B(x)=$ $x^{2} F(x)=x^{2-\xi / 2} f(x)$, we have

$$
x^{4} f(x) \frac{d^{2} \phi}{d x^{2}}+x^{2}\left[\left(2-\frac{\xi}{2}\right) x f(x)+x^{2} \frac{d f(x)}{d x}+2 i \omega x^{\xi / 2}\right] \frac{d \phi}{d x}-\left[\frac{\xi}{2} x^{2} f(x)-x^{3} \frac{d f(x)}{d x}+l(l+1) x^{2}\right] \phi=0,
$$

which can be rewritten as

$$
S(x) \frac{d^{2} \phi}{d x^{2}}+\frac{T(x)}{x-x_{+}} \frac{d \phi}{d x}+\frac{U(x)}{\left(x-x_{+}\right)^{2}} \phi=0,
$$

with

$$
\begin{aligned}
& S(x)=-\frac{x^{4} f(x)}{\left(x-x_{+}\right)}, \\
& T(x)=-x^{2}\left[\left(2-\frac{\xi}{2}\right) x f(x)+x^{2} \frac{d f(x)}{d x}+2 i \omega x^{\xi / 2}\right], \\
& U(x)=\left(x-x_{+}\right)\left[\frac{\xi}{2} x^{2} f(x)-x^{3} \frac{d f(x)}{d x}+l(l+1) x^{2}\right] .
\end{aligned}
$$

We can expand $S(x), T(x)$ and $U(x)$ around the point $x=x_{+}$and the corresponding coefficients of $\left(x-x_{+}\right)^{m}$ are described by $S_{m}, T_{m}$ and $U_{m}$, respectively. Since there exists only the ingoing modes near the event horizon, we can write $\phi$ as

$$
\phi=\sum_{k=0}^{\infty} a_{k}\left(x-x_{+}\right)^{k} .
$$


Substituting Eq. (16) into Eq. (14), we can obtain a recursion relation for $a_{n}$ :

$$
a_{n}=-\frac{1}{Z_{n}} \sum_{k=0}^{n-1}\left[k(k-1) S_{n-k}+k T_{n-k}+U_{n-k}\right] a_{k}
$$

where $Z_{n}=n(n-1) S_{0}+n T_{0}$. The boundary condition (12) at infinity now becomes

$$
\sum_{k=0}^{\infty} a_{k}\left(-x_{+}\right)^{k}=0
$$

In order to find the numerical solution of Eq. (18), we have to truncate the sum (18) at some large $k=N$. For chosen parameters, if the root of Eq. (18) approaches to the same value as $N$ increases, we can say this root is the characteristic frequencies of the black hole. Moreover, we must increase the precision of all the input data to avoid the "noise" which arises in the recursion.

\section{NUMERICAL RESULTS}

In this section we present our numerical results for the scalar perturbation in the Hořava-Lifshitz black holes with $\lambda=\frac{1}{3}, \frac{1}{2}$ and 3 , respectively.

\section{A. Hořava-Lifshitz black hole with $\lambda=1 / 3$}

In table II, we list the fundamental characteristic frequencies of the scalar perturbation in the HoravaLifshitz black holes with $\lambda=\frac{1}{3}$ for different values of $r_{+}$. From the table II, we find that in this black hole the real parts of frequencies vanish for the perturbations. This means that the scalar perturbations decay without any oscillation. As the radius of the event horizon $r_{+}$increase, the absolute values of imaginary parts increase. It implies that the scalar perturbations decay more quickly for the larger Hořava-Lifshitz black hole. Moreover, we find that the relation between $-\omega_{I}^{\lambda=1 / 3}$ and the temperature $T_{H}^{\lambda=1 / 3}$ can be approximated as

$$
-\omega_{I}^{\lambda=1 / 3}=7.54 T_{H}^{\lambda=1 / 3}
$$

which is also shown in Fig. (2). This means that the absolute value of imaginary parts is proportional to the Hawking temperature of the black hole. It is similar to that in 4-dimensional Schwarzschild AdS black hole in which the imaginary parts of the massless scalar perturbation can be approximated as [33]

$$
-\omega_{I}^{\mathrm{SAdS}}=11.16 T_{H}^{\mathrm{SAdS}}
$$

Comparing eqs. (19) and (20) we find that, if the Hawking temperature is identical, the scalar perturbation decays in the Hořava-Lifshitz black hole with $\lambda=1 / 3$ more slowly than that in the Schwarzschild AdS black 


\begin{tabular}{cccc}
\hline \hline$r_{+}$ & $\omega(l=0)$ & $\omega(l=1)$ & $\omega(l=2)$ \\
\hline 100 & $-175909790 \mathrm{i}$ & $-175911432 \mathrm{i}$ & $-175914714 \mathrm{i}$ \\
50 & $-10995509 \mathrm{i}$ & $-10995919 \mathrm{i}$ & $-10996740 \mathrm{i}$ \\
10 & $-17651.551 \mathrm{i}$ & $-17667.974 \mathrm{i}$ & $-17700.788 \mathrm{i}$ \\
2 & $-30.594857 \mathrm{i}$ & $-31.260342 \mathrm{i}$ & $-32.560976 \mathrm{i}$ \\
1.5 & $-10.284618 \mathrm{i}$ & $-10.662441 \mathrm{i}$ & $-11.389131 \mathrm{i}$ \\
1 & $-2.3735090 \mathrm{i}$ & $-2.5451323 \mathrm{i}$ & $-2.8627931 \mathrm{i}$ \\
0.8 & $-1.1145043 \mathrm{i}$ & $-1.2262111 \mathrm{i}$ & $-1.4267695 \mathrm{i}$ \\
0.4 & $-0.1443857 \mathrm{i}$ & $-0.1740443 \mathrm{i}$ & $-0.2214635 \mathrm{i}$ \\
0.1 & $-0.0064607 \mathrm{i}$ & $-0.0083985 \mathrm{i}$ & $-0.0111964 \mathrm{i}$ \\
0.04 & $-0.0010114 \mathrm{i}$ & $-0.0013222 \mathrm{i}$ & $-0.0017677 \mathrm{i}$ \\
$1 / 30$ & $-0.0007015 \mathrm{i}$ & $-0.0009173 \mathrm{i}$ & $-0.0012266 \mathrm{i}$ \\
0.02 & $-0.0002520 \mathrm{i}$ & $-0.0003298 \mathrm{i}$ & $-0.0004410 \mathrm{i}$ \\
0.01 & $-0.0000629 \mathrm{i}$ & $-0.0000824 \mathrm{i}$ & $-0.0001102 \mathrm{i}$ \\
\hline
\end{tabular}

TABLE II: The fundamental $(n=0)$ purely damped frequencies of scalar field in the in the $\lambda=1 / 3$ Hořava-Lifshitz black hole for $l=0,1,2$.
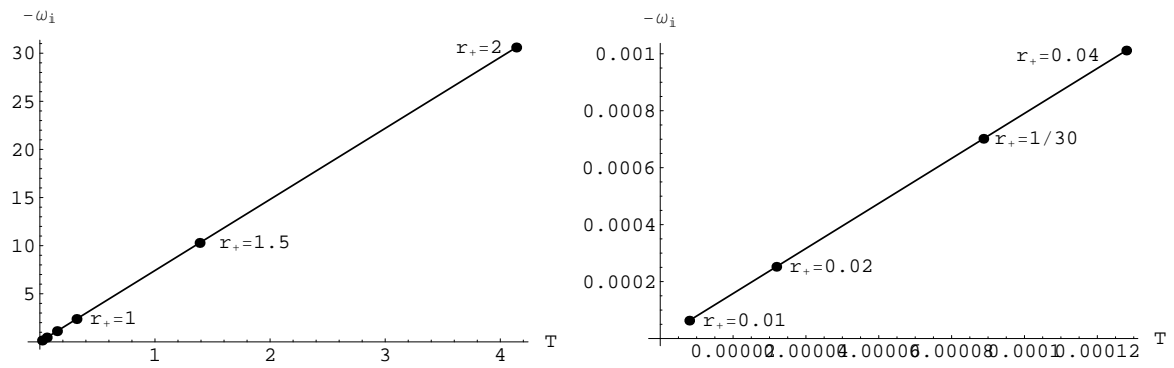

FIG. 2: For Hor̆ava-Lifshitz black hole with $\lambda=1 / 3,-\omega_{I}^{\lambda=1 / 3}$ is proportional to the temperature. The figure is for $l=0$ case.

hole. There are other different properties compared to Schwarzschild AdS black hole. On one hand, the damped modes appear to increase with the angular momentum $l$, while for Schwarzschild AdS black hole the imaginary part of the frequencies decrease with $l$ [33]. On the other hand, the linear relation between the imaginary part of the frequencies and temperature is broken down for small Schwarzschild AdS black hole, but to $\lambda=1 / 3$ Hořava-Lifshitz black hole, this relation holds not only for large and intermediate black holes, but also for small black hole (see Fig. 2).

We also study the asymptotic behavior of high overtone of the large, intermediate and small $\lambda=1 / 3$ Hořava-Lifshitz black hole. In the table (III)-(V), we list the overtones of purely damped frequencies of scalar field for fixed $l=0$, and 4 in the Hořava-Lifshitz black hole with $\lambda=1 / 3$ for $r_{+}=100, r_{+}=1$ and $r_{+}=0.01$, respectively. As the overtone number $n$ increases, the absolute value of imaginary parts increase and the modes are evenly spaced, which is similar to that in the usual black-hole spacetimes. In the large Hořava-Lifshitz black hole with $\lambda=1 / 3$ (see table III), the evenly spaced frequencies of the perturbations is 


\begin{tabular}{crcccc}
\hline \hline$n$ & $\omega(l=0)$ & $\omega(l=4)$ & $n$ & $\omega(l=0)$ & $\omega(l=4)$ \\
\hline & & & & & \\
0 & $-175909790 \mathrm{i}$ & $-175926204 \mathrm{i}$ & 8 & $-1384766991 \mathrm{i}$ & $-1384773476 \mathrm{i}$ \\
1 & $-329818196 \mathrm{i}$ & $-329830731 \mathrm{i}$ & 9 & $-1534973359 \mathrm{i}$ & $-1534979534 \mathrm{i}$ \\
2 & $-481590494 \mathrm{i}$ & $-481601084 \mathrm{i}$ & 10 & $-1685148584 \mathrm{i}$ & $-1685154489 \mathrm{i}$ \\
3 & $-632632175 \mathrm{i}$ & $-632641529 \mathrm{i}$ & 11 & $-1835299632 \mathrm{i}$ & $-1835305301 \mathrm{i}$ \\
4 & $-783328595 \mathrm{i}$ & $-783337071 \mathrm{i}$ & 12 & $-1985431502 \mathrm{i}$ & $-1985436962 \mathrm{i}$ \\
5 & $-933832036 \mathrm{i}$ & $-933839846 \mathrm{i}$ & 13 & $-2135547882 \mathrm{i}$ & $-2135553153 \mathrm{i}$ \\
6 & $-1084215699 \mathrm{i}$ & $-1084222980 \mathrm{i}$ & 14 & $-2285651553 \mathrm{i}$ & $-2285656654 \mathrm{i}$ \\
7 & $-1234519450 \mathrm{i}$ & $-1234526298 \mathrm{i}$ & 15 & $-2435744657 \mathrm{i}$ & $-2435749604 \mathrm{i}$ \\
\hline
\end{tabular}

TABLE III: The overtones of purely damped frequencies of scalar field in the Hořava-Lifshitz black hole with $\lambda=1 / 3$ for $r_{+}=100, l=0,4$. The asymptotic behavior is $\omega^{\lambda=1 / 3}=-(1.506 n+1.767) r_{+}^{4} i$ for any value of $l$.

\begin{tabular}{cccccc}
\hline \hline$n$ & $\omega(l=0)$ & $\omega(l=4)$ & $n$ & $\omega(l=0)$ & $\omega(l=4)$ \\
\hline & & & & & \\
0 & $-2.373509 \mathrm{i}$ & $-3.778299 \mathrm{i}$ & 9 & $-20.491285 \mathrm{i}$ & $-21.171191 \mathrm{i}$ \\
1 & $-4.428416 \mathrm{i}$ & $-5.624912 \mathrm{i}$ & 10 & $-22.492943 \mathrm{i}$ & $-23.145882 \mathrm{i}$ \\
2 & $-6.451969 \mathrm{i}$ & $-7.515653 \mathrm{i}$ & 11 & $-24.494323 \mathrm{i}$ & $-25.123273 \mathrm{i}$ \\
3 & $-8.465115 \mathrm{i}$ & $-9.433003 \mathrm{i}$ & 12 & $-26.495486 \mathrm{i}$ & $-27.102915 \mathrm{i}$ \\
4 & $-10.473494 \mathrm{i}$ & $-11.367757 \mathrm{i}$ & 13 & $-28.496477 \mathrm{i}$ & $-29.084457 \mathrm{i}$ \\
5 & $-12.479287 \mathrm{i}$ & $-13.314594 \mathrm{i}$ & 14 & $-30.497331 \mathrm{i}$ & $-31.067620 \mathrm{i}$ \\
6 & $-14.483518 \mathrm{i}$ & $-15.270211 \mathrm{i}$ & 15 & $-32.498073 \mathrm{i}$ & $-33.052181 \mathrm{i}$ \\
7 & $-16.486736 \mathrm{i}$ & $-17.232437 \mathrm{i}$ & 16 & $-34.498723 \mathrm{i}$ & $-35.037955 \mathrm{i}$ \\
8 & $-18.489259 \mathrm{i}$ & $-19.199784 \mathrm{i}$ & 17 & $-36.499293 \mathrm{i}$ & $-37.024791 \mathrm{i}$ \\
\hline
\end{tabular}

TABLE IV: The overtones of purely damped frequencies of scalar field in the Hořava-Lifshitz black hole with $\lambda=1 / 3$ for $r_{+}=1, l=0,4$. For large $n$ one finds that $\omega^{\lambda=1 / 3} \sim-(2.007 n+2.906) i$ for $l=0$.

\begin{tabular}{cccccc}
\hline \hline$n$ & $\omega(l=0)$ & $\omega(l=4)$ & $n$ & $\omega(l=0)$ & $\omega(l=4)$ \\
\hline & & & & & \\
0 & $-0.0000629 \mathrm{i}$ & $-0.0001721 \mathrm{i}$ & 9 & $-0.0005206 \mathrm{i}$ & $-0.0006121 \mathrm{i}$ \\
1 & $-0.0001158 \mathrm{i}$ & $-0.0002199 \mathrm{i}$ & 10 & $-0.0005708 \mathrm{i}$ & $-0.0006616 \mathrm{i}$ \\
2 & $-0.0001672 \mathrm{i}$ & $-0.0002682 \mathrm{i}$ & 11 & $-0.0006210 \mathrm{i}$ & $-0.0007111 \mathrm{i}$ \\
3 & $-0.0002182 \mathrm{i}$ & $-0.0003168 \mathrm{i}$ & 12 & $-0.0006712 \mathrm{i}$ & $-0.0007607 \mathrm{i}$ \\
4 & $-0.0002688 \mathrm{i}$ & $-0.0003657 \mathrm{i}$ & 13 & $-0.0007213 \mathrm{i}$ & $-0.0008104 \mathrm{i}$ \\
5 & $-0.0003193 \mathrm{i}$ & $-0.0004147 \mathrm{i}$ & 14 & $-0.0007715 \mathrm{i}$ & $-0.0008600 \mathrm{i}$ \\
6 & $-0.0003698 \mathrm{i}$ & $-0.0004639 \mathrm{i}$ & 15 & $-0.0008216 \mathrm{i}$ & $-0.0009097 \mathrm{i}$ \\
7 & $-0.0004201 \mathrm{i}$ & $-0.0005132 \mathrm{i}$ & 16 & $-0.0008717 \mathrm{i}$ & $-0.0009594 \mathrm{i}$ \\
8 & $-0.0004704 \mathrm{i}$ & $-0.0005626 \mathrm{i}$ & 17 & $-0.0009218 \mathrm{i}$ & $-0.0010092 \mathrm{i}$ \\
\hline
\end{tabular}

TABLE V: The overtones of purely damped frequencies of scalar field in the Hořava-Lifshitz black hole with $\lambda=1 / 3$ for $r_{+}=0.01, l=0,4$. For large $n$ one finds that $\omega^{\lambda=1 / 3} \sim-(5.05 n+6.33) \times 10^{-5} i$ for $l=0$.

approximated as

$$
\omega^{\lambda=1 / 3}=-\frac{4 \pi}{3}(1.506 n+1.767) T_{H}^{\lambda=1 / 3} i
$$

This asymptotic behavior for the spacing holds for any value of $l$, i.e. this spacing is $l$-independent as the same as Schwarzschild AdS black hole [36]. 


\section{B. Hor̆ava-Lifshitz black hole with $\lambda=\frac{1}{2}$}

Now let us consider the scalar perturbation in the Hořava-Lifshitz black hole with $\lambda=1 / 2$. In table VI, we list the fundamental characteristic frequencies of the scalar perturbation for different values of $r_{+}$. Similarly, we find that in this black hole the real parts of frequencies also disappear for the perturbations (as shown in Fig. (3) ). Moreover, we also find the absolute values of imaginary parts increase with the radius of the event horizon $r_{+}$. It implies that in this case the scalar perturbations decay more quickly in the larger black hole. For the Hořava-Lifshitz black hole with $\lambda=\frac{1}{2}$, the relation between $-\omega_{I}^{\lambda=1 / 2}$ and the Hawking temperature

\begin{tabular}{cccc}
\hline \hline$r_{+}$ & $\omega(l=0)$ & $\omega(l=1)$ & $\omega \quad(l=2)$ \\
\hline & & & \\
100 & $-13333.333 \mathrm{i}$ & $-13333.666 \mathrm{i}$ & $-13334.333 \mathrm{i}$ \\
50 & $-3333.3333 \mathrm{i}$ & $-3333.6666 \mathrm{i}$ & $-3334.3333 \mathrm{i}$ \\
10 & $-133.33333 \mathrm{i}$ & $-133.66666 \mathrm{i}$ & $-134.33333 \mathrm{i}$ \\
5 & $-33.333333 \mathrm{i}$ & $-33.666666 \mathrm{i}$ & $-34.333333 \mathrm{i}$ \\
1 & $-1.333333 \mathrm{i}$ & $-1.666666 \mathrm{i}$ & $-2.333333 \mathrm{i}$ \\
0.8 & $-0.853333 \mathrm{i}$ & $-1.186666 \mathrm{i}$ & $-1.853333 \mathrm{i}$ \\
0.6 & $-0.480000 \mathrm{i}$ & $-0.813333 \mathrm{i}$ & $-1.464000 \mathrm{i}$ \\
0.4 & $-0.213333 \mathrm{i}$ & $-0.546666 \mathrm{i}$ & $-0.977142 \mathrm{i}$ \\
\hline
\end{tabular}

TABLE VI: The fundamental $(n=0)$ purely damped frequencies of scalar field in the in the $\lambda=\frac{1}{2}$ Hořava-Lifshitz black hole for fixed $l=0,1,2$.

reads approximately

$$
-\omega_{I}^{\lambda=1 / 2}=8.378 T_{H}^{\lambda=1 / 2}
$$

This relation holds not only for large and intermediate black holes, but also for small black hole. Comparing it with Eq.(20), we find, as the Hawking temperature is identical, the scalar perturbation decays more slowly in the Hořava-Lifshitz black hole with $\lambda=1 / 2$. Similarly, in the table (VII)-(IX), we list the overtones of purely
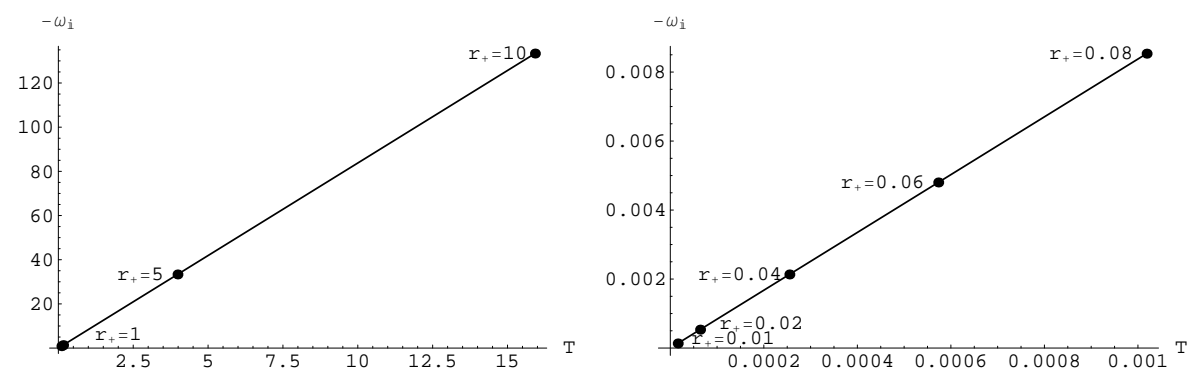

FIG. 3: For $\lambda=1 / 2$ Hořava-Lifshitz black hole, $-\omega_{I}$ is proportional to the Hawking temperature. The figure is for $l=0$ case.

damped frequencies of scalar field for fixed $l=0$ and $l=4$ in the Hořava-Lifshitz black hole with $\lambda=1 / 2$ for $r_{+}=100, r_{+}=1$ and $r_{+}=0.4$, respectively. We find that the modes are evenly spaced for large black hole 
(see table VII)

$$
\omega^{\lambda=1 / 2}=-2 \pi(1.009 n+1.333) T_{H}^{\lambda=1 / 2} i
$$

This asymptotic behavior for the spacing holds for any value of $l$, i.e. this spacing is also $l$-independent as the same as $\lambda=1 / 3$ Hořava-Lifshitz black hole.

\begin{tabular}{cccccc}
\hline \hline$n$ & $\omega(l=0)$ & $\omega(l=4)$ & $n$ & $\omega(l=0)$ & $\omega(l=4)$ \\
\hline & & & & & \\
0 & $-13333.333 \mathrm{i}$ & $-13336.666 \mathrm{i}$ & 9 & $-104761.90 \mathrm{i}$ & $-104762.38 \mathrm{i}$ \\
1 & $-24000.000 \mathrm{i}$ & $-24002.000 \mathrm{i}$ & 10 & $-114782.60 \mathrm{i}$ & $-114783.04 \mathrm{i}$ \\
2 & $-34285.714 \mathrm{i}$ & $-34287.142 \mathrm{i}$ & 11 & $-124800.00 \mathrm{i}$ & $-124800.40 \mathrm{i}$ \\
3 & $-44444.444 \mathrm{i}$ & $-44445.555 \mathrm{i}$ & 12 & $-134814.81 \mathrm{i}$ & $-134815.18 \mathrm{i}$ \\
4 & $-54545.454 \mathrm{i}$ & $-54546.363 \mathrm{i}$ & 13 & $-144827.58 \mathrm{i}$ & $-144827.93 \mathrm{i}$ \\
5 & $-64615.384 \mathrm{i}$ & $-64616.153 \mathrm{i}$ & 14 & $-154838.70 \mathrm{i}$ & $-154839.03 \mathrm{i}$ \\
6 & $-74666.666 \mathrm{i}$ & $-74667.333 \mathrm{i}$ & 15 & $-164848.48 \mathrm{i}$ & $-164848.78 \mathrm{i}$ \\
7 & $-84705.882 \mathrm{i}$ & $-84706.470 \mathrm{i}$ & 16 & $-174857.14 \mathrm{i}$ & $-174857.42 \mathrm{i}$ \\
8 & $-94736.842 \mathrm{i}$ & $-94737.368 \mathrm{i}$ & 17 & $-184864.86 \mathrm{i}$ & $-184865.13 \mathrm{i}$ \\
\hline
\end{tabular}

TABLE VII: The overtones of purely damped frequencies of scalar field in the Hořava-Lifshitz black hole with $\lambda=1 / 2$ for $r_{+}=100, l=0,4$. For large black hole one finds approximately $\omega^{\lambda=1 / 2}=-(1.009 n+1.333) r_{+}^{2} i$ for any value of $l$.

\begin{tabular}{cccccc}
\hline \hline$n$ & $\omega(l=0)$ & $\omega(l=4)$ & $n$ & $\omega(l=0)$ & $\omega(l=4)$ \\
\hline & & & & & \\
0 & $-1.333333 \mathrm{i}$ & $-4.400000 \mathrm{i}$ & 9 & $-10.47619 \mathrm{i}$ & $-10.95238 \mathrm{i}$ \\
1 & $-2.400000 \mathrm{i}$ & $-4.666666 \mathrm{i}$ & 10 & $-11.47826 \mathrm{i}$ & $-11.91304 \mathrm{i}$ \\
2 & $-3.428571 \mathrm{i}$ & $-4.857142 \mathrm{i}$ & 11 & $-12.48000 \mathrm{i}$ & $-12.88000 \mathrm{i}$ \\
3 & $-4.444444 \mathrm{i}$ & $-5.555555 \mathrm{i}$ & 12 & $-13.48148 \mathrm{i}$ & $-13.85185 \mathrm{i}$ \\
4 & $-5.454545 \mathrm{i}$ & $-6.363636 \mathrm{i}$ & 13 & $-14.48275 \mathrm{i}$ & $-14.82758 \mathrm{i}$ \\
5 & $-6.461538 \mathrm{i}$ & $-7.230769 \mathrm{i}$ & 14 & $-15.48387 \mathrm{i}$ & $-15.80645 \mathrm{i}$ \\
6 & $-7.466666 \mathrm{i}$ & $-8.133333 \mathrm{i}$ & 15 & $-16.48484 \mathrm{i}$ & $-16.78787 \mathrm{i}$ \\
7 & $-8.470588 \mathrm{i}$ & $-9.058823 \mathrm{i}$ & 16 & $-17.48571 \mathrm{i}$ & $-17.77142 \mathrm{i}$ \\
8 & $-9.473684 \mathrm{i}$ & $-10.00000 \mathrm{i}$ & 17 & $-18.48648 \mathrm{i}$ & $-18.75675 \mathrm{i}$ \\
\hline
\end{tabular}

TABLE VIII: The overtones of purely damped frequencies of scalar field in the Hořava-Lifshitz black hole with $\lambda=1 / 2$ for $r_{+}=1, l=0,4$. Asymptotically for large $n$ one finds approximately $\omega^{\lambda=1 / 2} \sim-(1.009 n+1.333) i$ for $l=0$.

\begin{tabular}{cccccc}
\hline \hline$n$ & $\omega(l=0)$ & $\omega(l=4)$ & $n$ & $\omega(l=0)$ & $\omega(l=4)$ \\
\hline & & & & & \\
0 & $-0.213333 \mathrm{i}$ & $-1.781818 \mathrm{i}$ & 9 & $-1.676190 \mathrm{i}$ & $-2.384000 \mathrm{i}$ \\
1 & $-0.384000 \mathrm{i}$ & $-1.803076 \mathrm{i}$ & 10 & $-1.836521 \mathrm{i}$ & $-2.396800 \mathrm{i}$ \\
2 & $-0.548571 \mathrm{i}$ & $-1.82222 \mathrm{i}$ & 11 & $-1.996800 \mathrm{i}$ & $-2.527407 \mathrm{i}$ \\
3 & $-0.711111 \mathrm{i}$ & $-1.861333 \mathrm{i}$ & 12 & $-2.157037 \mathrm{i}$ & $-2.662068 \mathrm{i}$ \\
4 & $-0.872727 \mathrm{i}$ & $-1.943529 \mathrm{i}$ & 13 & $-2.317241 \mathrm{i}$ & $-2.800000 \mathrm{i}$ \\
5 & $-1.033846 \mathrm{i}$ & $-1.977142 \mathrm{i}$ & 14 & $-2.477419 \mathrm{i}$ & $-2.940606 \mathrm{i}$ \\
6 & $-1.194666 \mathrm{i}$ & $-2.042105 \mathrm{i}$ & 15 & $-2.637575 \mathrm{i}$ & $-3.083428 \mathrm{i}$ \\
7 & $-1.355294 \mathrm{i}$ & $-2.152380 \mathrm{i}$ & 16 & $-2.797714 \mathrm{i}$ & $-3.228108 \mathrm{i}$ \\
8 & $-1.515789 \mathrm{i}$ & $-2.271304 \mathrm{i}$ & 17 & $-2.957837 \mathrm{i}$ & $-3.374358 \mathrm{i}$ \\
\hline
\end{tabular}

TABLE IX: The overtones of purely damped frequencies of scalar field in the Hořava-Lifshitz black hole with $\lambda=1 / 2$ for $r_{+}=0.4, l=0,4$. Asymptotically for large $n$ one finds approximately $\omega^{\lambda=1 / 2} \sim-(0.161 n+0.221) i$ for $l=0$. 


\section{Hor̆ava-Lifshitz black hole with $\lambda=3$}

For $\lambda=3$, there exists extremal case with $r_{+}=1$ because the temperature vanishes. It is thermodynamically unstable even for large black hole [26] and shows some marginal properties. For example, the small black holes $\left(r_{+}<1\right)$ have no physical meaning since the temperature is negative, while for large black holes the temperature approach to a constant $T_{H}^{\lambda=3} \rightarrow \frac{1}{4 \pi}$. Therefore the decay of scalar perturbation will different from previous cases $\lambda=1 / 3$ and $\lambda=1 / 2$.

By repeating the previous operation, we find that it is very difficult to find the convergent root. Under the condition of 100-digital precision of input data, there is no convergent root. So we improve precision to 300-digital precision, the convergent roots appear but the convergence becomes much slower than $\lambda=1 / 3$ and $\lambda=1 / 2$ cases. It is also difficult to seek high overtone.

\begin{tabular}{cccc}
\hline \hline$r_{+}$ & $\omega(l=0)$ & $r_{+}$ & $\omega(l=0)$ \\
\hline & & & \\
100 & $-70.252656 \mathrm{i}$ & 4 & $-2.353367 \mathrm{i}$ \\
50 & $-34.897542 \mathrm{i}$ & 3 & $-1.630615 \mathrm{i}$ \\
10 & $-6.611997 \mathrm{i}$ & 2 & $-0.880420 \mathrm{i}$ \\
5 & $-3.067646 \mathrm{i}$ & & \\
\hline
\end{tabular}

TABLE X: The fundamental $(n=0)$ damped frequencies of scalar field in the Hořava-Lifshitz black hole with $\lambda=3$ for $l=0$.
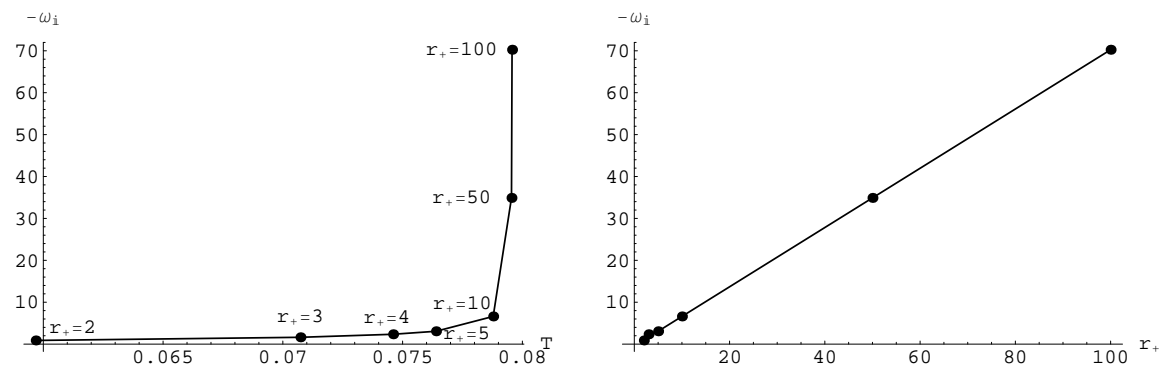

FIG. 4: The purely damped modes for the scalar perturbations in the Hořava-Lifshitz black hole with $\lambda=3$ and $l=0$. Though the modes do not linear to the temperature (left), they linear to the radii of events horizon, i.e., $-\omega_{I}^{\lambda=3}=0.669 r_{+}($right $)$.

In the table $(\mathrm{X})$ we list the characteristic frequencies of the scalar perturbation in the Hořava-Lifshitz black holes with $\lambda=3$ for different values of $r_{+}$. From the table $(\mathrm{X})$, it is easy to see that the modes are not linear to the temperature at all, but they are still monotonically increasing with the temperature (see Fig. (44)). Though the modes do not linear to the temperature, they linear to the radii of horizon in large black hole region

$$
-\omega_{I}^{\lambda=3}=0.669 r_{+}
$$


This property is similar to Schwarzschild AdS black hole, $-\omega_{I}^{S A d S}=2.66 r_{+}[33]$.

\section{CONCLUSIONS AND DISCUSSIONS}

We study the dynamical evolution of the massless scalar perturbation in the background of Hořava-Lifshitz black-hole spacetime. For the cases of dynamical coupling constants $\lambda=1 / 3,1 / 2$ and 3 , there are purely damped modes which are different from those in the usual black-hole spacetimes. For $\lambda=1 / 3$ and $1 / 2$ cases the imaginary parts of frequency are proportional to the Hawking temperature not only for large and intermediate black holes, but also for small black hole. If the black holes possess the same Hawking temperatures, the scalar perturbation decays most quickly in the Schwarzschild AdS black-hole spacetime. For $\lambda=3$ case, the imaginary parts of frequency are not proportional to the Hawking temperature any more, but is linear to the radii of event horizon of the black hole.

Why there are only purely damped modes in three kinds of Hořava-Lifshitz black holes? Let us recall that purely damped mode is also appeared in Einstein's gravity, for example, some modes of the Schwarzschild AdS black hole due to Dirac [35], electromagnetic and gravitational [36, 37] perturbations and Reissner-Nordström AdS black hole due to electromagnetic, gravitational 38] and Rarita-Schwinger [39] field perturbations for low overtone numbers. But for scalar field, the purely damped mode has never appeared in Einstein's gravity. In classical mechanics, the over-damped condition will lead to a purely damped mode. By comparing the wave equation (10) to damped wave equation in classical mechanics, we find that the potential $V(r)$ is exact damped term which results in energy dissipation. From the Fig. (11), one can see that the potential of scalar perturbation for $\lambda=1 / 3$ Hořava-Lifshitz black hole is proportional to $r^{8}$ at long distance, for $\lambda=1 / 2$ HořavaLifshitz black hole it is $r^{4}$, but for Schwarzschild AdS black hole is just $r^{2} \cdot r^{4}$ and $r^{8}$ asymptotic potentials may be over-damped potentials, so that the decay with no oscillation for $\lambda=1 / 3$ and $\lambda=1 / 2$ Hořava-Lifshitz black holes.

From the Fig. (5) we know that the potential of electromagnetic perturbation with $l=1$ in Schwarzschild AdS black hole is convergent to constant 2 at infinity and potential of odd gravitational perturbation with $l=2$ is 6 [36, 37], while for $\lambda=3$ Hořava-Lifshitz black hole it converges to constant 1 at infinity (Fig. (10). The authors in Ref. [37] find that, due to electromagnetic perturbation, there exist the lowest purely damped modes so long as $r_{+} \geq 5$ with angular momentum $l=1$, and $r_{+} \geq 10$ with $l=2$; to odd gravitational perturbation, there exist the lowest purely damped modes if only $r_{+} \geq 0.5$ with $l=2$, and $r_{+} \geq 1$ with $l=3$. In ref. [36], the authors find that there exist eight purely damped modes for $r_{+}=1000$, four for $r_{+}=100$, and two for 
$r_{+}=10$. Then they conclude that "infinitely large black holes may have pure imaginary electromagnetic QN frequencies for any overtone number". Therefore, it is understandable that there exist purely damped modes in $\lambda=3$ Hořava-Lifshitz black hole.
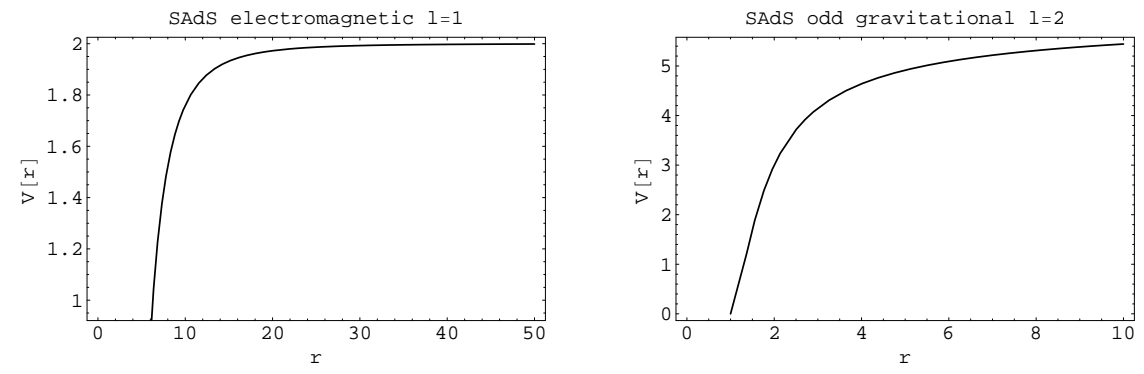

FIG. 5: The potential for the electromagnetic perturbation with $l=1, r_{+}=5$ (left), and for the odd gravitational perturbation with $l=2, r_{+}=1$ (right) in the Schwarzschild AdS black hole.

\section{Acknowledgments}

This work was supported by the National Natural Science Foundation of China under Grant No 10875040; the FANEDD under Grant No. 200317, the Hunan Provincial Natural Science Foundation of China under Grant No. 08JJ0001, the Hunan Provincial Innovation Foundation for Postgraduate, and Construct Program of the National Key Discipline.

[1] P. Horava, Phys. Rev. D 79084008 (2009), arXiv:0901.3775 [hep-th]].

[2] P. Horava, JHEP 0903020 (2009), arXiv:0812.4287] [hep-th]].

[3] P. Hořava, Phys. Rev. Lett. 102161301 (2009), arXiv:0902.3657 [hep-th].

[4] A. Volovich and C. Wen, JHEP 0905:087 (2009), arXiv: 0903.2455 [hep-th].

[5] J. Kluson, JHEP 0907:079 (2009), arXiv:0904.1343 [hep-th].

[6] H. Nikolic, arXiv:0904.3412 [hep-th].

[7] H. Nastase, arXiv:0904.3604 [hep-th].

[8] K. I. Izawa, arXiv:0904.3593 [hep-th].

[9] G. E. Volovik, Pis'ma ZhETF 89 627, JETP. Lett 89525 (2009), arXiv:0904.4113 [gr-qc].

[10] B. Chen and Q. G. Huang, arXiv:0904.4565 [hep-th].

[11] R. G. Cai, B. Hu and H. B. Zhang, Phys. Rev. D 80, 041501 (2009), arXiv:0905.0255 [hep-th].

[12] G. Calcagni, arXiv:0904.0829 [hep-th].

[13] T. Takahashi and J. Soda, Phys. Rev. Lett. 102231301 (2009), arXiv:0904.0554 [hep-th].

[14] S. Mukohyama, JCAP 0906:001 (2009), arXiv:0904.2190 [hep-th].

[15] R. Brandenberger, arXiv:0904.2835 [hep-th].

[16] Y. S. Piao, arXiv:0904.4117 [hep-th].

[17] X. Gao, arXiv:0904.4187 [hep-th].

[18] E. Kiritsis and G. Kofinas, to be appeared in Nucl. Phys. B, arXiv:0904.1334 [hep-th].

[19] E. O. Colgain and H. Yavartanoo, JHEP 0908:021 (2009), arXiv:0904.4357 [hep-th].

[20] H. Lu, J. Mei and C. N. Pope, Phys. Rev. Lett. 103, 091301 (2009), arXiv:0904.1595 [hep-th].

[21] R. G. Cai, L. M. Cao and N. Ohta, Phys. Rev. D 80024003 (2009), arXiv:0904.3670 [hep-th].

[22] R. G. Cai, Y. Liu and Y. W. Sun, JHEP 0906:010 (2009), arXiv:0904.4104 [hep-th].

[23] A. Ghodsi, arXiv:0905.0836 [hep-th].

[24] Y. S. Myung and Y. W. Kim, arXiv:0905.0179 [hep-th].

[25] T. Nishioka, arXiv:0905.0473 [hep-th]. 
[26] R. G. Cai, L. M. Cao and N. Ohta, Phys. Lett. B 679504 (2009), arXiv:0905.0751 [hep-th].

[27] Y. S. Myung, to be appeared in PLB, arXiv:0905.0957 [gr-qc]].

[28] S. B Chen and J. L. Jing, Phys. Rev. D 80024036 (2009), arXiv: 0905.2055.

[29] R. A. Konoplya, Phys. Lett. B 679499 (2009), arXiv: 0905.1523.

[30] S. B. Chen and J. L. Jing, arXiv: 0905.1409.

[31] T. Harko, Z. Kovacs and F. S. N. Lobo, Phys. Rev. D 80, 044021 (2009), arXiv:0907.1449

[32] R. L. Arnowitt, S. Deser and C.W. Misner, The dynamics of general relativity, "Gravitation: an introduction to current research", Louis Witten ed. (Wilew 1962), chapter 7, pp 227-265, arXiv:gr-qc/0405109.

[33] G. T. Horowitz and V. E. Hubery, Phys. Rev. D 62024027 (2000).

[34] J. Shen, B. Wang, R. K. Su, C. Y. Lin and R. G. Cai, J. High Energy Phys. 07037 (2007).

[35] M. Giammatteo and J. L. Jing, Phys. Rev. D 71024007 (2005).

[36] V. Cardoso, R. Konoplya and J. P. S. Lemos, Phys. Rev. D 68044024 (2003).

[37] V. Cardoso and J. P. S. Lemos, Phys. Rev. D 64084017 (2001).

[38] E. Berti and K. D. Kokkotas, Phys. Rev. D 67064020 (2003).

[39] Y. Zhang and J. L. Jing, Int. J. Mod. Phys. D 15905 (2005). 